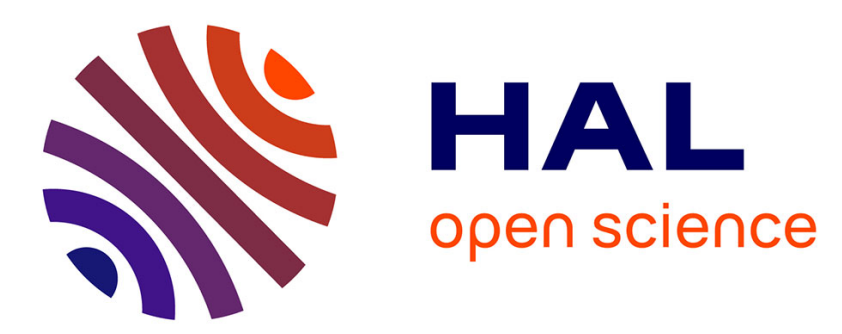

\title{
Interband absorption in aluminium under pressure
}

\author{
M. Alouani, M.A. Khan
}

\section{To cite this version:}

M. Alouani, M.A. Khan. Interband absorption in aluminium under pressure. Journal de Physique, 1986, 47 (3), pp.453-460. 10.1051/jphys:01986004703045300 . jpa-00210225

\section{HAL Id: jpa-00210225 https://hal.science/jpa-00210225}

Submitted on 1 Jan 1986

HAL is a multi-disciplinary open access archive for the deposit and dissemination of scientific research documents, whether they are published or not. The documents may come from teaching and research institutions in France or abroad, or from public or private research centers.
L'archive ouverte pluridisciplinaire HAL, est destinée au dépôt et à la diffusion de documents scientifiques de niveau recherche, publiés ou non, émanant des établissements d'enseignement et de recherche français ou étrangers, des laboratoires publics ou privés. 


\title{
Interband absorption in aluminium under pressure
}

\author{
M. Alouani and M. A. Khan \\ Laboratoire de Magnétisme et de Structure Electronique des Solides $\left({ }^{*}\right)$, \\ Université Louis Pasteur, 4, rue Blaise Pascal, 67070 Strasbourg Cedex, France
}

(Reçu le 29 juillet 1985, révisé le 18 octobre 1985, accepté le 25 octobre 1985)

\begin{abstract}
Résumé. - A partir du schéma de bandes ab initio et autocohérentes, nous présentons les premières études théoriques de propriétés optiques d'aluminium sous pression hydraulique. En accord avec les expériences, nous observons les déplacements des pics d'absorption en fonction des pressions. Nous mettons en évidence les changements respectifs dans les structures de bandes pour expliquer ces déplacements.
\end{abstract}

\begin{abstract}
The first theoretical study of optical absorption in aluminium under hydraulic pressure, based on a self-consistent $a b$ initio band scheme, is presented. The shifts of the absorption peaks under high pressure, in agreement with the experimental data, are observed. The relevant changes in the band structures are pointed out to explain these shifts.
\end{abstract}

\section{Introduction.}

During the last two decades the optical properties of Aluminium under normal pressure have been extensively studied [1-17].

Recently, Tups and Syassen [17] have studied the optical reflectivity of aluminium (Al) under high pressure in the range of $300 \mathrm{kbar}$ at room temperature. They observed a shift of the reflection peaks towards the higher energies as a function of increasing pressure. These shifts are satisfactorily explained on the basis of the corresponding changes in the pseudopotentials $U(111)$ and $U(200)$. The peak positions are simply twice of these pseudopotentials. In this approach a very fundamental assumption is that the $\mathrm{Al}$ crystal retains its nearly free electron nature even at very high pressure.

Our aim, in the present work, is to study the optical properties of $\mathrm{Al}$ at high pressure using a self-consistent $a b$ initio band structure. For this purpose we use the method of Linear Muffin-Tin Orbitals(LMTO) $[18,19]$ with spherical correction to calculate the energy bands. No adjustable parameters are introduced. The exchange correlation is taken care of by the local density theory [20]. The pressure effect is introduced by the corresponding changes in the crystal volume.

In the second section, we present the band structures at different pressures and, then (Sections 3 and 4 ) these

(*) U.A. au CNRS no 306. band schemies are used to calculate respectively the electronic pressure and the imaginary part of the interband dielectric function $\varepsilon_{2}^{\mathrm{b}}(\omega)$. In section 5 , we shall discuss and compare our results with those obtained experimentally and the last section will conclude the paper.

\section{Band structure.}

The method of the Linear-Muffin-Tin Orbitals has become such a common feature these days that we shall not describe it here. The theoretical and practical aspects of the method are presented in detail elsewhere [18-19]. We consider a frozen core and treat $3 \mathrm{~s}$, $3 p$ and $3 d$ as band electrons. Once the energy bands with spherical correction are obtained we use only the atomic sphere orbitals of the proper function to calculate the dipole matrix elements.

It is well-established that $\mathrm{Al}$ retains its fcc crystal structure even under high pressure [15, 17]. The band structures are obtained for $\mathrm{Al}$ in fcc phase as a function of volume changes corresponding to different pressures. The self-consistency is attained after about fifteen iterations. The detailed band scheme and the density of states are calculated with a fine mesh of 946 points in the 1/48th Brillouin Zone (BZ).

Figures 1 and 2 show the band schemes obtained at ordinary pressure (i.e. crystal volume $=V_{0}$ ) and under a pressure where the crystal volume $V=0.8 V_{0}$. The corresponding densities of states are presented in figures 3 and 4 . Figures 1 and 2 clearly show the effects 

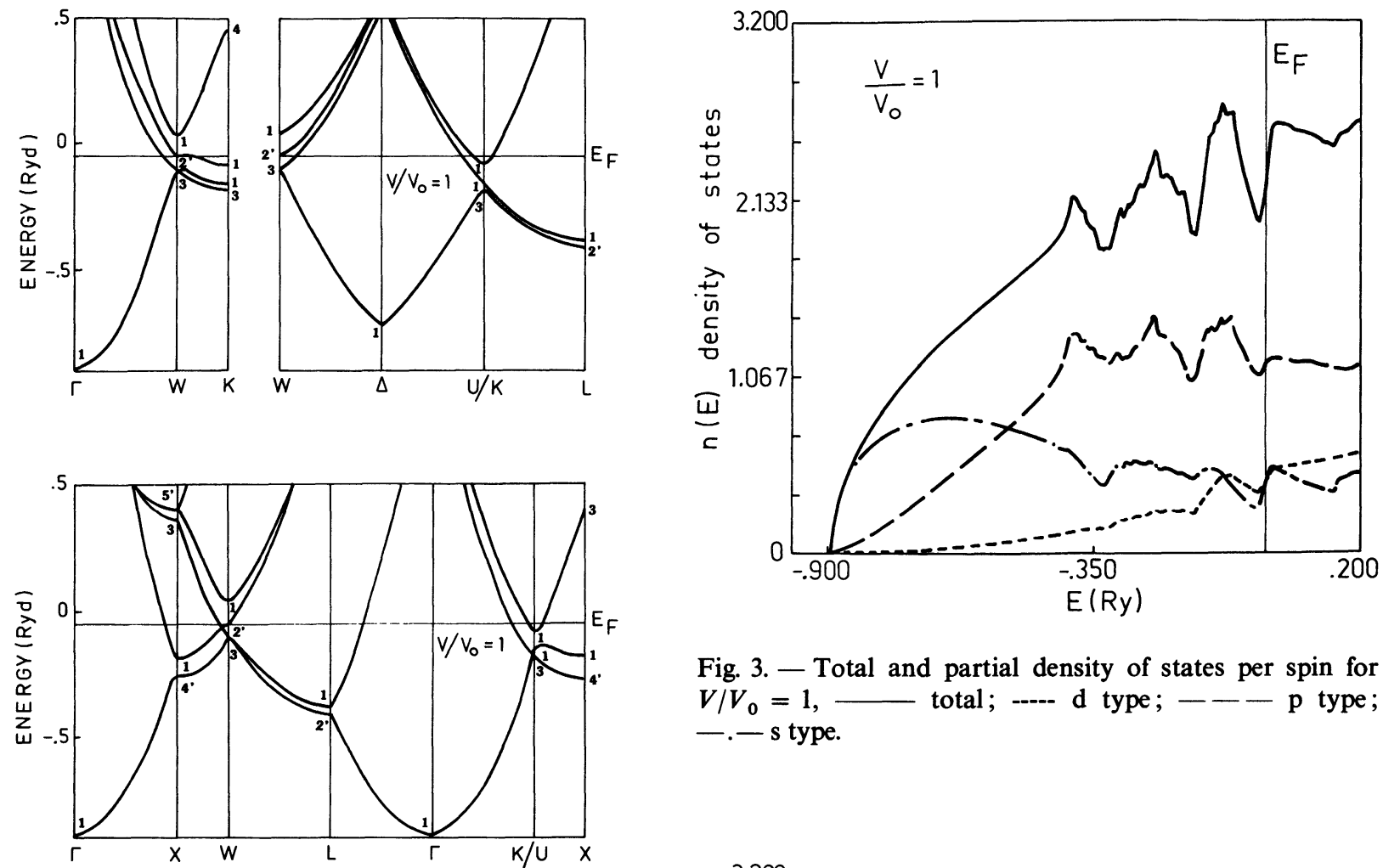

Fig. 3. - Total and partial density of states per spin for $V / V_{0}=1$, $\longrightarrow$ total; -..- d type; - - $\mathrm{p}$ type; -. - s type.

Fig. 1. - Electronic structure of aluminium for $V / V_{0}=1$; $\Delta$ is the Mid-point of $\Gamma-\mathrm{X}$.
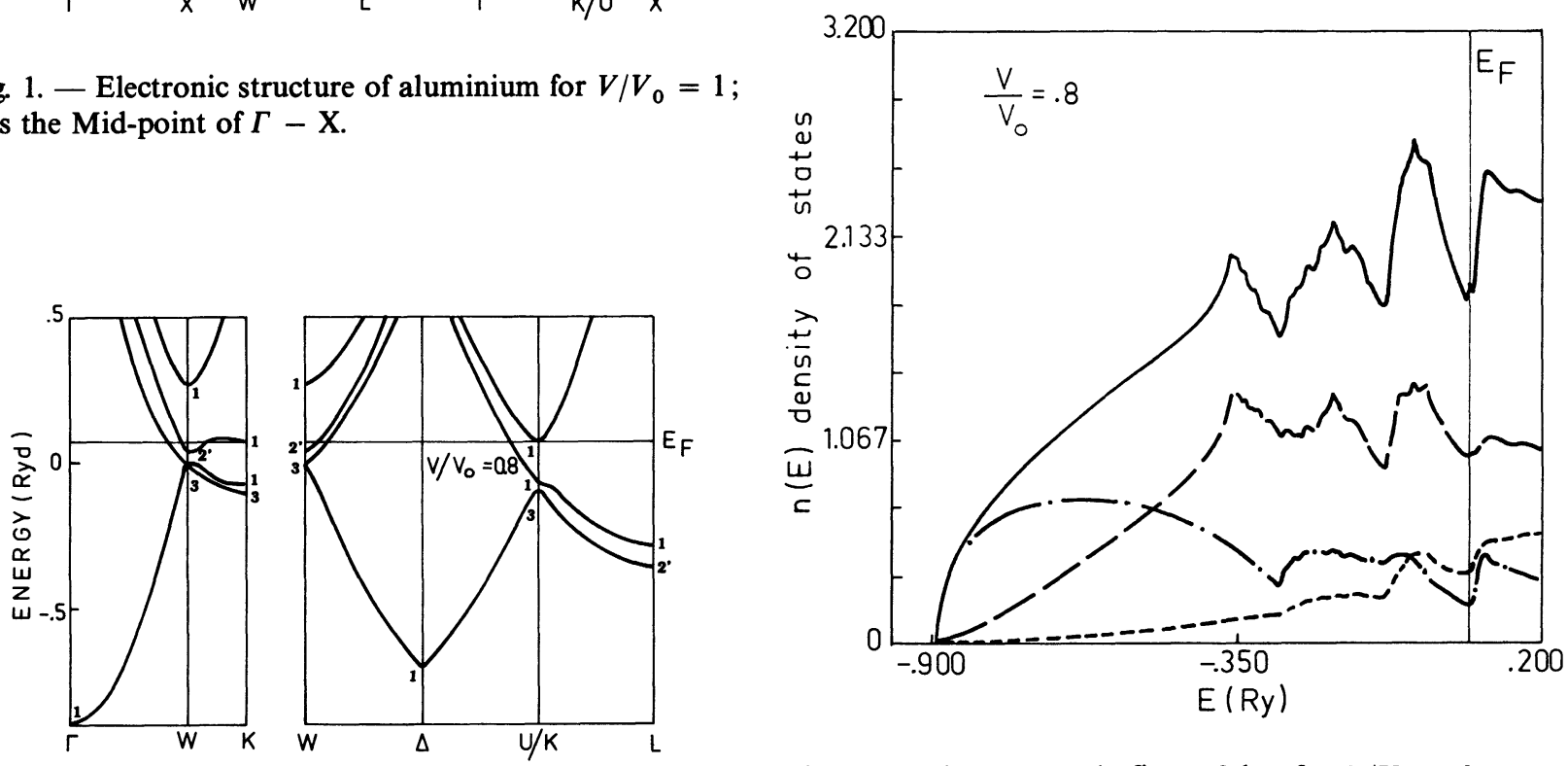

Fig. 4. - The same as in figure 3 but for $V / V_{0}=0.8$.

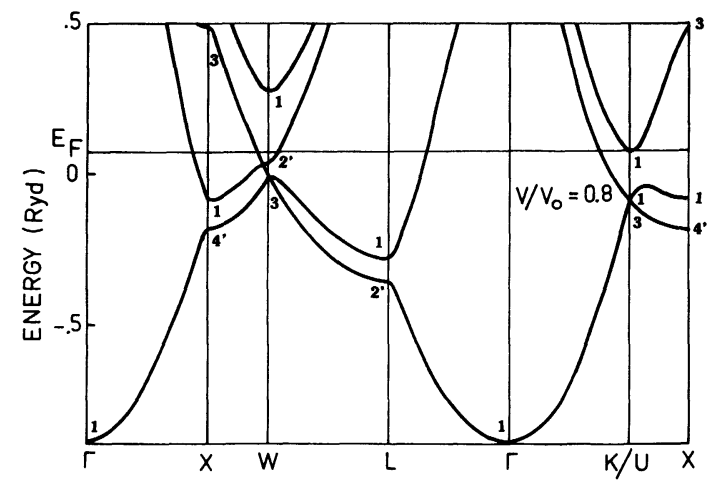

of pressure on the parallel bands where the interband transitions take place. The partial density of states (Fig. 3 and 4) reveals the importance of the delectrons around the Fermi level and hence their participation in band to band transitions at low energy. A comparative study of the present band structure with other theoretical $[12,13]$ and experimental $[21]$ schemes is presented in table $I$. We shall discuss its main features in section 5. In table II we give some physical quantities as a function of $V / V_{0}$. From this table one can easily conclude that :

Fig. 2. - Electronic structure of aluminium for $V / V_{0}=0.8$.

i) Though the total number of « d » electrons $\left(N_{\mathrm{d}}\right)$ 
Table $\mathrm{I}$. - Energies (in $\mathrm{eV}$ ) at some high symmetry points in $\mathrm{Al} . E_{\mathrm{F}}$ is the reference level.

\begin{tabular}{lccccc}
\multicolumn{1}{c}{ Symmetry } & \multicolumn{2}{c}{ Present work } & Self-consistent & APW & Experimental \\
& $V / V_{0}=1$ & $V / V_{0}=0.8$ & LCGO [12] & {$[13]$} & values [21] \\
\hline$\Gamma_{1}$ (band width) & -11.41 & -13.10 & -11.10 & -11.05 & $-10.6 \pm 0.2$ \\
$\mathrm{X}_{1}$ & -1.92 & -2.10 & -1.64 & -1.77 & $-1.15 \pm 0.04$ \\
$\mathrm{X}_{4}^{\prime}$ & -2.94 & -3.44 & -2.96 & -2.76 & $-2.83 \pm 0.04$ \\
Gap at X & 1.02 & 1.34 & 1.32 & 0.99 & $1.68 \pm 0.08$ \\
(i.e. $\left.\mathrm{X}_{1}-\mathrm{X}_{4}^{\prime}\right)$ & -4.85 & -5.79 & -4.64 & -4.53 & -4.55 \\
$\mathrm{~L}_{2}^{\prime}$ & & & & &
\end{tabular}

Table II. - For a crystal volume V at high pressure as compared to volume $V_{0}$ with crystal constant « a » $=7.62 \mathrm{a}$.u, a certain number of physical quantities are presented. $E_{\mathrm{F}}$ is the Fermi level. $N_{\mathrm{i}}$ is the total number of $\mathrm{i}$ type electrons below the Fermi level, $n_{i}\left(E_{\mathrm{F}}\right)$ is the $i$-th partial density of states at the Fermi level, $P V$ is product of the pressure $P$ and volume $V, P V_{\mathrm{i}}(\mathrm{i}=\mathrm{s}, \mathrm{p}, \mathrm{d})$ is the partial $P V .\left[N_{\mathrm{i}}, n_{i}\left(E_{\mathrm{F}}\right)\right.$ and $P V_{\mathrm{i}}$ are given for one spin. $]$

\begin{tabular}{ccccccccccccr}
$V / V_{\mathbf{0}}$ & $E_{\mathrm{F}}(\mathrm{Ry})$ & $N_{\mathrm{s}}$ & $N_{\mathrm{p}}$ & $N_{\mathrm{d}}$ & $n_{\mathbf{s}}\left(E_{\mathrm{F}}\right)$ & $n_{\mathrm{p}}\left(E_{\mathrm{F}}\right)$ & $n_{\mathrm{d}}\left(E_{\mathrm{F}}\right)$ & $P V(\mathrm{Ry})$ & $P V_{\mathbf{s}}(\mathrm{Ry})$ & $P V_{\mathrm{p}}(\mathrm{Ry})$ & $P V_{\mathrm{d}}(\mathrm{Ry})$ & $P(\mathrm{kbar})$ \\
\hline 1 & -0.0478 & 0.5652 & 0.7239 & 0.2108 & 0.5772 & 1.2835 & 0.8089 & -0.0236 & 0.0541 & -0.0214 & -0.0445 & -31.624 \\
0.9 & 0.0049 & 0.5349 & 0.7405 & 0.2246 & 0.4506 & 1.1573 & 0.8071 & 0.0393 & 0.0589 & 0.0029 & -0.0423 & 58.504 \\
0.8 & 0.0713 & 0.5093 & 0.7514 & 0.2392 & 0.1990 & 0.9745 & 0.6600 & 0.1435 & 0.0713 & 0.0377 & -0.0372 & 240.446 \\
0.7 & 0.1564 & 0.4863 & 0.7587 & 0.2550 & 0.1679 & 0.8865 & 0.6503 & 0.2979 & 0.0916 & 0.0864 & -0.0290 & 570.446 \\
0.6 & 0.2718 & 0.4639 & 0.7636 & 0.2725 & 0.1342 & 0.8009 & 0.6302 & 0.5290 & 0.1225 & 0.1577 & -0.0157 & 1181.857
\end{tabular}

in the occupied states is small $(14 \%)$ yet around the Fermi level the d states $n_{\mathrm{d}}\left(E_{\mathrm{F}}\right)$ are very important.

ii) The d electrons become more and more important as we increase the pressure.

Under these observations we insist that for any theoretical study of the optical absorption, it is necessary to include $p \rightleftharpoons d$ transitions. The observable changes in the Fermi surface of $\mathrm{Al}$ are illustrated in figure 5 where the Fermi surface contours in the high symmetry plane [100] for $V / V_{0}=1$ and 0.8 are presented.

\section{Electronic pressure.}

For the calculation of the electronic pressure $P$ at $0 \mathrm{~K}$, we use the formula derived by Nieminen and Hodges [22] and Pettifor [23] from the virial expression given by Liberman [24] :

$3 P V=2 \sum_{l} \int^{E_{\mathrm{F}}} \mathrm{d} E n_{l}(E) S \phi_{l}^{2}(E, S)\left(\frac{-d D_{1}(E)}{d \ln S}\right)$

where :

$$
\begin{aligned}
\frac{-d D_{1}(E)}{d \ln S}=\left(D_{1}(E)+\right. & l+1)\left(D_{1}(E)-l\right)+ \\
& +\left(E-V(S)-\mathrm{E}_{\mathrm{xc}}+V_{\mathrm{xc}}\right) S^{2}
\end{aligned}
$$

and :

$$
D_{l}(E)=\left.\frac{S}{\phi_{l}(E, S)} \frac{\partial \phi_{l}(E, r)}{\partial r}\right|_{r=S}
$$

$V$ is the volume of the unit cell, $S$ is the atomic sphere radius. $D_{l}(E)$ is the logarithmic derivative of the radial wave function $\phi_{l}(E, r)$ at the cell boundary. $V(S)$ is the potential at $r=S . V_{\mathrm{xc}}$ is the exchange correlation potential [20] and $E_{x c}$ is the exchange correlation energy density obtained from homogeneous electron gas.

The electronic pressures obtained for different volumes are given in table II. Figure 6 shows these pressures in comparison to other experimental and theoretical results.

Usually the zero-point vibrational corrections are ignored which is certainly true when the crystal is under high compression but at normal volume it may not be so. The easiest way to include this correction is to use the Debye model $[25,26]$ where the zeropoint energy $E_{0}$ is :

$$
E_{0}=\frac{9}{8} k_{\mathrm{B}} \theta_{\mathrm{D}}(\mathrm{Ry} / \text { atom })
$$

and the corresponding pressure is :

$$
P_{0}=\gamma E_{0} / V
$$

$k_{\mathrm{B}}$ is the Boltzmann constant, $\theta_{\mathrm{D}}$ the Debye temperature, $\gamma$ the Grünseissen constant and $V$ the cell volume. Following Janak et al. [26] the zero-point pressure at volume $V=V_{0}$ is calculated in the present case. We obtain $P_{0}=8.94 \mathrm{kbar}$. As compared to the electronic pressure $-31.624 \mathrm{kbar}$, the zero-point pressure is of opposite sign and roughly $1 / 3$ in magnitude. Adding this contribution does not change the overall picture and moreover it is calculated through an empirical procedure with a measured Debye temperature. 

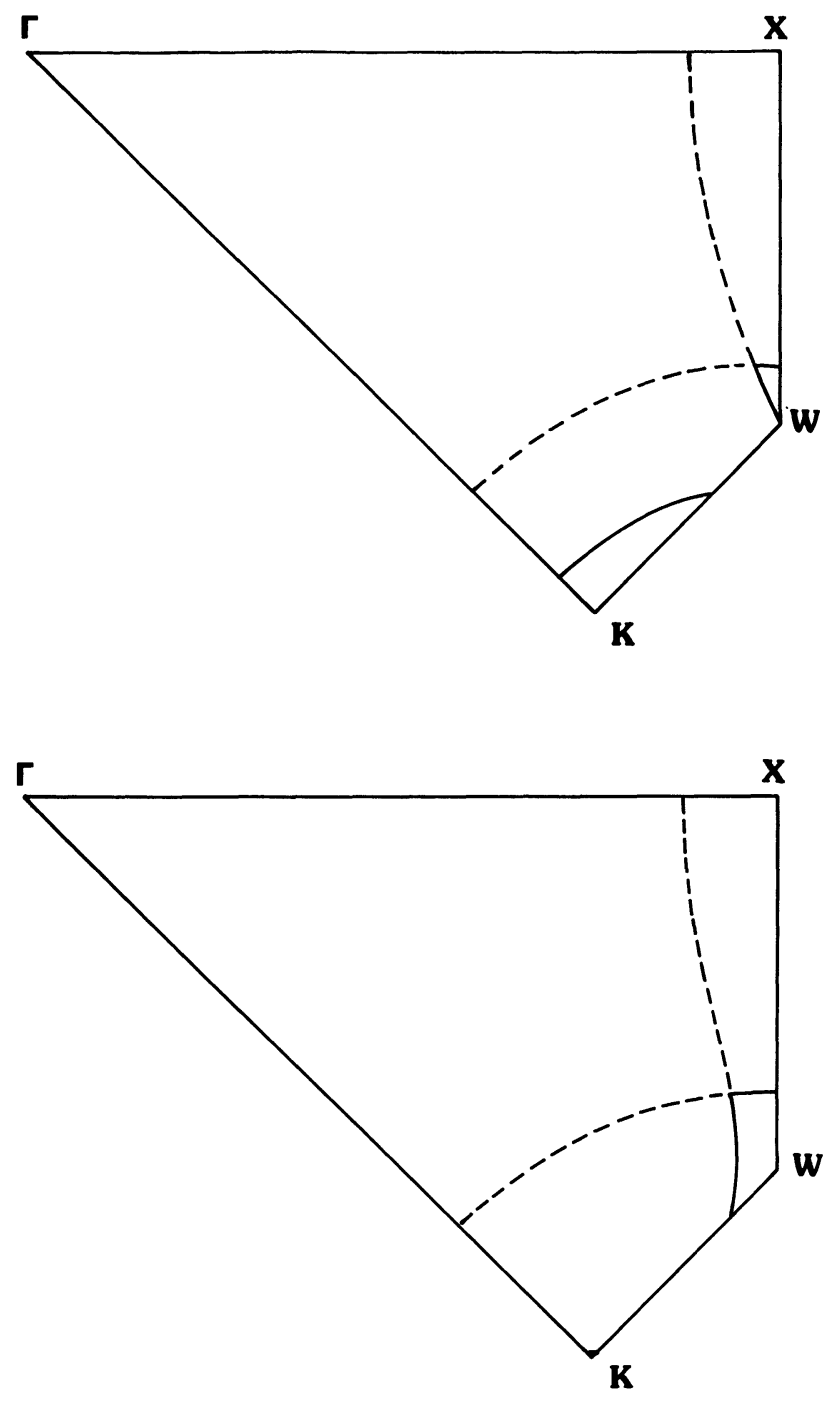

Fig. 5. - The Fermi surface contours of $\mathrm{Al}$ at normal pressure (a) and at $V / V_{0}=0.8(\mathrm{~b})$. The full and the dashed lines indicate $3 \mathrm{rd}$ and 2 nd band contours respectively.

Hence we shall limit ourselves to the electronic pressure in our further discussions.

\section{Optical absorption.}

The imaginary part $\varepsilon_{2}(\omega)$ of the dielectric function, for a given photon energy $\omega$ (in atomic units $\hbar=1$, $e^{2}=1 / m=2$ ), is the sum of two contributions.

(i) The free charge carriers or free electrons give an important effect at low energy limit which is also called Drude's term and then

(ii) the interband transitions give rise to structures in the optical absorption curves :

$$
\varepsilon_{2}(\omega)=\varepsilon_{2}^{\mathrm{f}}(\omega)+\varepsilon_{2}^{\mathrm{b}}(\omega)
$$

where the free electron $\varepsilon_{2}^{\mathrm{f}}(\omega)$ is [1] :

$\varepsilon_{2}^{\mathrm{f}}(\omega)=\frac{4 \pi N_{\mathrm{f}} \mathrm{e}^{2}}{m^{*}} \frac{\tau_{\mathrm{f}}}{\omega\left(1+\omega^{2} \tau_{\mathrm{f}}^{2}\right)}=\frac{4 \pi \sigma_{0}}{\omega\left(1+\omega^{2} \tau_{\mathrm{f}}^{2}\right)}$

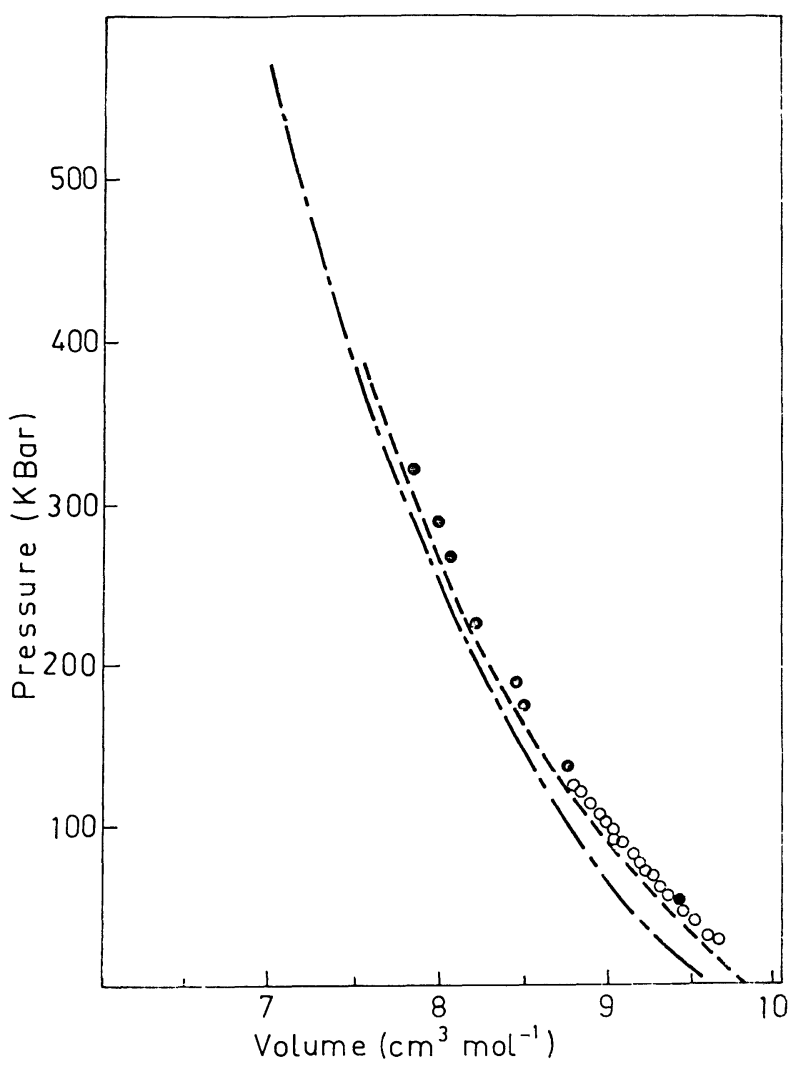

Fig. 6. - Pressure versus volume curve; - - - present work (--- Friedli and Ashcroft (Ref. [10]); open and closed circles from Ref. [17] and [31]).

$N_{\mathrm{f}}$ is the number of free charge carriers per unit volume. In metals, it is the number of s-p conduction electrons which can be determined through band structure. $m^{*}$ is the optical effective mass and it may also be determined from energy bands or more easily through the sum rules [4] (in a.u.) :

$$
\frac{1}{m^{*}}=\frac{1}{8 \pi^{2} N_{\mathrm{f}}} \int_{0}^{\infty} \omega \varepsilon_{2}^{\mathrm{f}}(\omega) \mathrm{d} \omega
$$

and :

$$
\frac{1}{m^{*}}=1-\frac{1}{8 \pi^{2} N_{\mathrm{f}}} \int_{0}^{\infty} \omega \varepsilon_{2}^{\mathrm{b}}(\omega) \mathrm{d} \omega
$$

The relaxation time $\tau_{\mathrm{f}}$ is determined from experiment $\mathbf{a l}$ measurements.

The interband contribution to the imaginary part of the dielectric function at $0 \mathrm{~K}$ (in a.u.) is :

$$
\varepsilon_{2}^{\mathrm{b}}(\omega)=\frac{1}{2 \pi \omega^{2}} \sum_{n} \sum_{n^{\prime}} \int_{\mathrm{S}} \frac{\left|P_{n n^{\prime}}(k)\right|^{2} \mathrm{~d} S}{\left|\nabla_{k} \omega_{n n^{\prime}}(k)\right|}
$$

where :

$$
\begin{gathered}
\omega_{n n^{\prime}}(k)=E_{n^{\prime}}(k)-E_{n}(k) \\
E_{n}(k) \leqslant E_{\mathrm{F}} \leqslant E_{n^{\prime}}(k) \\
S=\left\{\bar{k} ; \omega_{n n^{\prime}}(k)=\omega\right\}
\end{gathered}
$$

$\omega$ is the photon energy and $E_{\mathrm{F}}$ the Fermi level. 


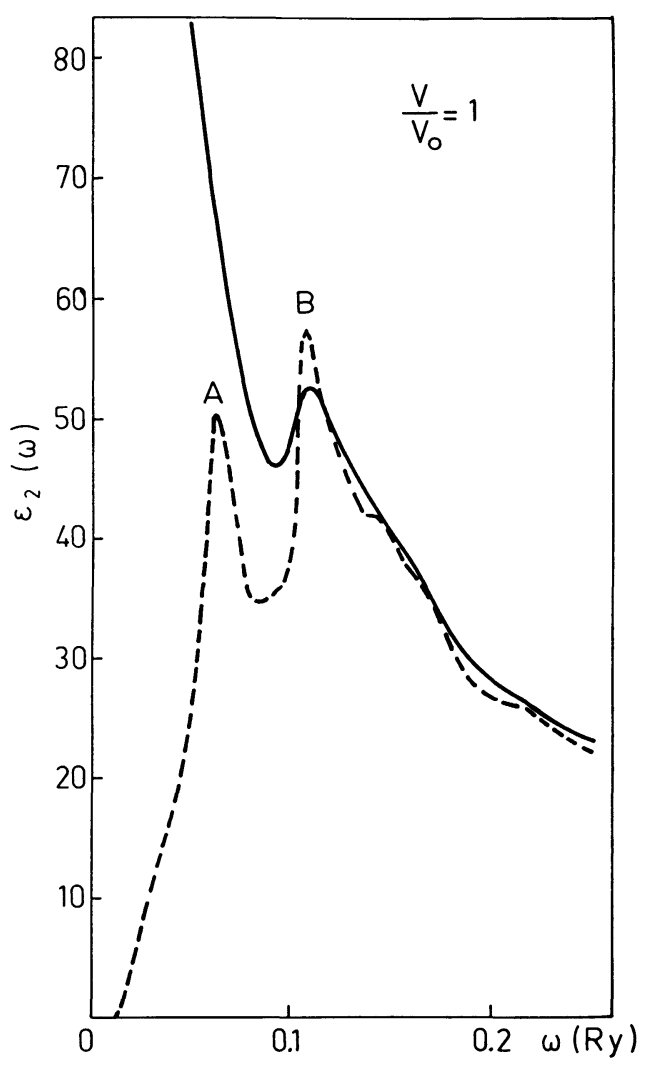

Fig. 7.

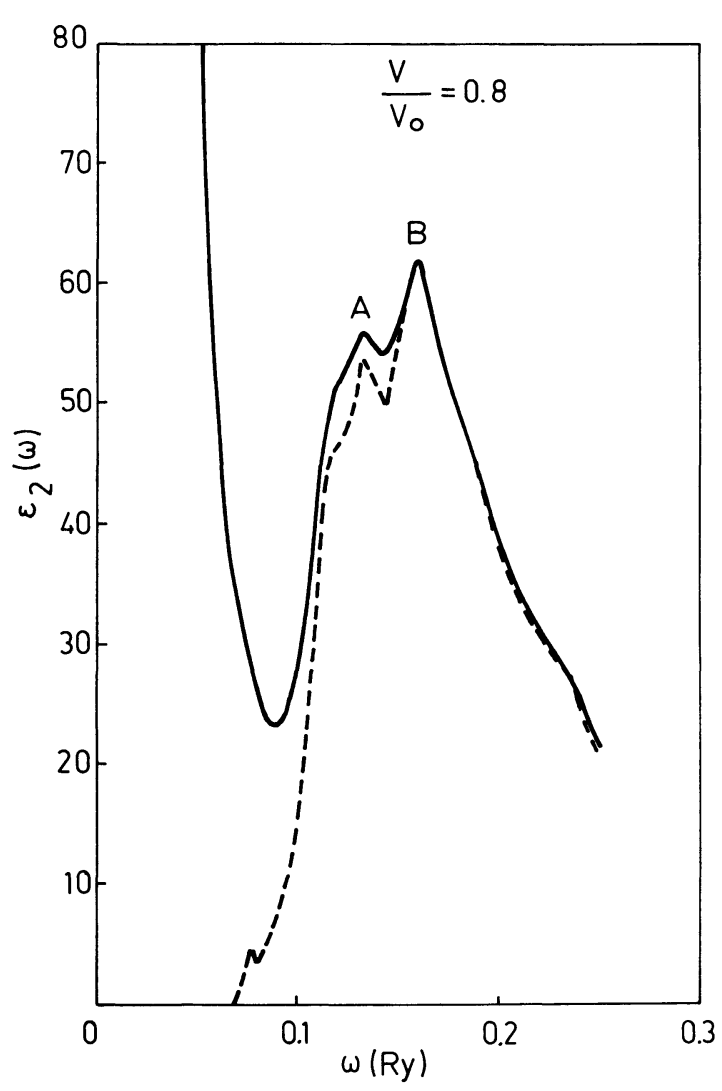

Fig. 8.

Figs. 7 and 8. - The total $\varepsilon_{2}(\omega)$ and interband $\varepsilon_{2}^{\mathrm{b}}(\omega)$ imaginary part of the dielectric constant for $V / V_{0}=1$ (Fig. 7) and $V / V_{0}=0.8$ (Fig. 8).

$$
P_{n n^{\prime}}(k)=\left\langle n k|P| n^{\prime} k\right\rangle
$$

$|n k\rangle$ is the proper function for the energy $E_{n}(k)$. The proper function $|n k\rangle$ when expressed in terms of atomic sphere approximation is very appropriate to calculate the dipole matrix elements $P_{n n^{\prime}}(k)$ [27]. The integration over the $\bar{k}$ space is performed by the tetrahedron method [28].

$\varepsilon_{2}^{\mathrm{b}}(\omega)$ is calculated in the low energy range (i.e. $\omega \leqslant 0.25 \mathrm{Ry}$ ). The results obtained are presented in figures 7 and 8 for $V / V_{0}=1$ and 0.8 respectively. In figure 9 we have plotted the positions of the two peaks thus calculated at different volumes and compare them with other experimental and theoretical data.

\section{Discussion.}

The electronic structures obtained by LMTO under different pressures give parallel bands intersecting the Fermi level. The comparison of the first two figures reveals the fact that the separations of the parallel bands change significantly when the crystal is subjected to a pressure. Though, from our numerical analysis, it is possible to obtain all the $\bar{k}$-points in the whole $\mathrm{BZ}$ which contribute to the two peaks in $\varepsilon_{2}^{\mathrm{b}}(\omega)$, but the major contributions to the peaks $A$ and $B$ at the lower and higher energy sides are due to the transitions between the second and the third bands following the directions $U-\Delta$ and $K-\Gamma$ respectively. The displacements of these peaks towards higher energy under pressure is evident from figures 1 and 2.

Figures 3 and 4 representing the total and partial density of states show that

i) under pressure the energy bands become wider and the separation between the high density of state regions is increased;

ii) there is a shift of the Fermi level $E_{\mathrm{F}}$ towards higher energy and

iii) the partial density of « $d$ » type states is quite appreciable, particularly around the Fermi level.

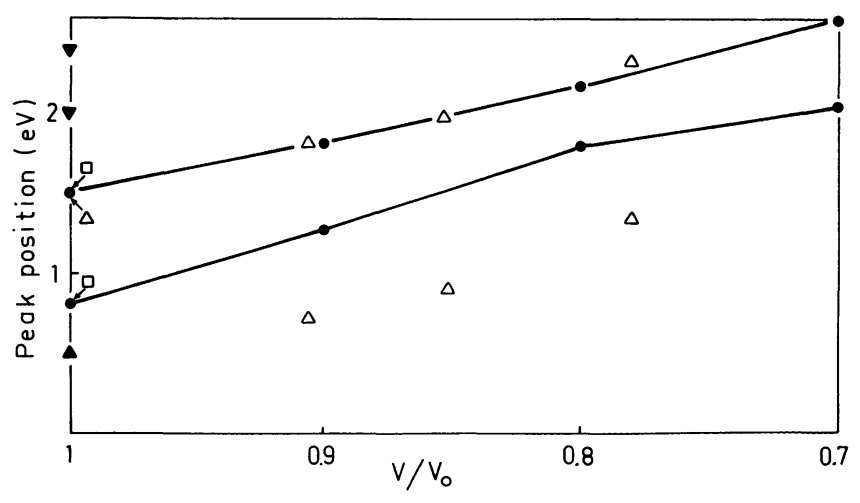

Fig. 9. $-\varepsilon_{2}^{\mathrm{b}}(\omega)$ peak positions versus volume; - present work; $\Delta$ Tups and Syassen (Ref. [17]); $\square$ Callaway and Laurent (Ref. [12]); $\Delta$ Bos and Lynch (Ref. [3]); $\boldsymbol{\nabla}$ Hunderi and Nilsson (Ref. [34]). 
From these observations one can easily predict that the absorption peaks will move towards higher energy under pressure as already stated previously. Moreover, the intensity of the absorption peaks should decrease at higher pressures and lastly the presence of " $d$ " electrons cannot be ignored for interband transitions.

The present calculation gives the occupied states band width (i.e. $E_{\mathrm{F}}-\Gamma_{1}$ ) as $11.41 \mathrm{eV}$ (Table I) which compares well with other theoretical values [12, 13] under normal pressure. A recent angle resolved photoemission experiment by Levinson et al. [21] shows that this width is rather $10.6 \pm 0.2 \mathrm{eV}$. The discrepancy between the measured and the theoretical values is mainly due to the absence of the non-local effects in any local density approximation for exchange and correlation potentials [21]. Another interesting value is the gap at critical point $X$ (i.e. $X_{1}-X_{4}^{\prime}$ ). We obtain $X_{1}-X_{4}^{\prime}=1.02 \mathrm{eV}$. It is very close to the one obtained by Szmulkowicz and Segall [13] but smaller than that obtained by Singhal and Callaway [12]. Comparing columns 2 and 3 of table I, one will notice that the band width $\left(E_{\mathrm{F}}-\Gamma_{1}\right)$ and the gap at the critical point $X$ increase when the pressure is applied.

In figure $5 \mathrm{a}$ and $\mathrm{b}$, we present the Fermi surface contours in [100] plane for normal volume and for $V / V_{0}=0.8$ respectively. The major contribution to the Fermi surface comes from the 2 nd band. At normal pressure there is also a small contribution near $W$ and a larger one around $K$ from the third band. These are often referred to as the third zone electron pockets and have been experimentally observed [29]. As the pressure is applied, the third zone electron pocket near $K$ shrinks and eventually disappears while that near $W$ grows (Fig. 5b). Tups and Syassen [17] have also obtained this particular behaviour with four-plane-wave-model. The present LMTO band calculation shows that the total area of the Fermi surface at $V / V_{0}=0.8$ is about $86 \%$ as compared to that at normal pressure. Hence, under pressure Al Fermi surface reveals some striking changes which have not been yet observed experimentally.

We have used the crystal parameter $" a$ " = 7.62 a.u. [30] which is the measured value at room temperature. At $0 \mathrm{~K}$ this parameter gives us a kind of inflated crystal and hence a negative pressure (Table II). It would be more realistic to obtain a crystal constant which gives a zero pressure at $0 \mathrm{~K}$, but this search will increase the calculation time considerably and will not give anything new in the relative behaviour of the band schemes at different pressures. Also, even with this enlarged crystal our calculated pressures at different volumes (Fig. 6) agree quite well with the experimental data $[17,31]$ at $300 \mathrm{~K}$ and the theoretical results at $0 \mathrm{~K}$ of Friedli and Ashcroft [10].

Figures 7 and 8 show the interband dielectric constants $\varepsilon_{2}^{\mathrm{b}}(\omega)$ and also the total $\varepsilon_{2}(\omega)$. Before adding $\varepsilon_{2}^{\mathrm{f}}(\omega)$ to $\varepsilon_{2}^{\mathrm{b}}(\omega)$ to obtain $\varepsilon_{2}(\omega)$ we have added a lifetime broadening as a Lorentzian to $\varepsilon_{2}^{\mathrm{b}}(\omega)$ to take the interband relaxation into account.

For the calculation of $\varepsilon_{2}^{f}(\omega)$ one needs to know many parameters and one of them is the optical effective mass $m^{*}$. Using the sum rule (7) we have calculated it at $V / V_{0}=1$ and 0.8 from calculated $\varepsilon_{2}^{\mathrm{b}}(\omega)$. Since the calculations have been performed for $\omega \leqq 0.25 \mathrm{Ry}$, we have added an exponential tail to $\varepsilon_{2}^{\mathrm{b}}(\omega)$ to take the contribution from the higher energies into account. We obtain the effective optical masses $m^{*}=2.32$ and 2.84 for $V / V_{0}=1$ and 0.8 respectively [17].

Though the calculated optical masses show an upward trend as a function of pressure, as observed experimentally [17], they are much greater than the corresponding experimental data. Tups and Syassen [17] obtained $m^{*}=1.45$ at normal pressure and 1.98 at $310 \mathrm{kbar}$ (i.e. $V / V_{0}=0.78=0.8$ ). This discrepancy requires some comments. It should be noted that the structure observed in $\varepsilon_{2}^{\mathrm{b}}(\omega)$ are local properties for some given photon energies $\omega$ whereas the optical mass is an integral quantity. Hence the agreement in peak positions and magnitudes does not necessarily entail the agreement of the optical masses.

Though we have a satisfactorily good agreement with the experimental peak positions we do not obtain a very rapidly decreasing $\varepsilon_{2}^{\mathrm{b}}(\omega)$ at higher energies as it is in experimental measurements. This slow decay of $\varepsilon_{2}^{\mathbf{b}}(\omega)$ beyond the peak B (Figs. 7 and 8) makes an important contribution and hence we obtain a very high optical mass. The reason of the slow decay of $\varepsilon_{2}^{b}(\omega)$ at higher energies in the present case are two.

- First, with the increase of the photon energies, the effect of $N$-electrons (which is not taken into consideration in one-electron band scheme) becomes more important,

- Second, at higher energies the atomic sphere approximation is not sufficient and it will be more appropriate to use full LMTO for the interband transitions.

The later point has been also raised by Uspenski et al. [32]. To test the merits of full LMTO over LMTO-ASA these authors have calculated the diagonal current density matrix at a fixed $\bar{k}$ point for copper in two cases. They obtain a better result in the first case and hence they conclude that the optical properties should be calculated with full LMTO. To prove the superiority of full LMTO for the optical properties it will be more convincing to use LMTO-ASA and full LMTO respectively and calculate the optical properties of a given solid because the diagonal current density matrix is only an intraband effect while the structures in the absorption curves are due to interband transitions. Moreover, the optical properties are global contributions in the whole $\bar{k}$ space and not a single $\bar{k}$ point effect. 
In a separate article [33] we have made this comparative study where we calculate the optical properties of $\mathrm{Al}$ and ferromagnetic iron with LMTO-ASA as well as with full LMTO. Thus we could show that for $\mathrm{Al}$ a better agreement with experimental observation in the high energy range is obtained when the full LMTO are used. On the other hand, to obtain the peak positions LMTO-ASA gives as good a result as full LMTO. Besides LMTO-ASA is three times faster. Under these circumstances, it seems to us that the ASA part of LMTO is sufficient to study the behaviour of two main absorption peaks of $\mathrm{Al}$ under pressure.

Since we have an appreciable discrepancy in our calculated optical masses in LMTO-ASA when compared to experimental values, we decided to calculate $\varepsilon_{2}^{f}(\omega)$ with the measured zero energy conductivity $\sigma_{0}$ and the free electron lifetime $\tau_{\mathrm{f}}$. At normal pressure (i.e. $V / V_{0}=1$ ) the experimental values given by Bennett and Bennett [2] are $\sigma_{0}=15.55 \mathrm{Ry}$ and $\tau_{\mathrm{f}}^{-1}=0.082 \mathrm{eV}$ and the corresponding values measured more recently by Tups and Syassen [17] are $12.62 \mathrm{Ry}$ and $0.09 \mathrm{eV}$ respectively, one can choose any one of these two sets, but in figure 7 we have included the Drude correction with the first set of parameters [2]. At higher pressure the only measurements are those of Tups and Syassen [17]. At $310 \mathrm{kbar}$ (i.e. $\left.V / V_{0}=0.780=0.8\right) \sigma_{0}=5.23 \mathrm{Ry}$ and $\tau_{\mathrm{f}}^{-1}=$ $0.18 \mathrm{eV}$. In figure 8 we have employed these values for the free electron term at $V / V_{0}=0.8$. For the Lorentzian broadening of the interband part $\varepsilon_{2}^{b}(\omega)$, the usual procedure is to choose a relaxation time $\tau$ which brings the calculated $\varepsilon_{2}^{b}(\omega)$ in agreement with the measured optical absorption. In the present case we have taken $\tau$ equal to $\tau_{\mathrm{f}}$ for $V / V_{0}=1$. Figure 6 (at $V / V_{0}=1$ ) shows clearly two peaks at $0.84 \mathrm{eV}$ (A) and $1.5 \mathrm{eV}$ (B) respectively. When we add the free electron (Drude correction) and the lifetime broadening, the lower energy peak A disappears and we are left with only $B$ which is the experimental situation in most measurements. With increasing pressure these two peaks are displaced towards the higher energy (Fig. 9) and they are broadened (Figs. 7 and 8). Looking at the interband part $\varepsilon_{2}^{b}(\omega)$ at $V / V_{0}=0.8$ (Fig. 8) we notice that a less broadening effect is required since the peaks are already much broader as compared to those in figure 7 . So, we chose arbitrarily $\tau^{-1}=0.03 \mathrm{eV}$. The aim is simply to see the effect of the relaxation time broadening and the Drude correction. We obtain an "observable" $A$ peak at $1.82 \mathrm{eV}$ due to the fact that now it falls into the energy range where the Drude corrections are not important. The positions of these two peaks versus volume are indicated in figure 9 . We obtain a very good agreement with the experimental measure- ments [17] at different pressures for peak B. Other measured or calculated values $[1-9,12,13]$ of $\mathbf{B}$ at $V / V_{0}=1$ are not very far from our result.

Unfortunately, the same cannot be said for peak $A$. When the free electron contribution is properly subtracted from the total optical data, a structure is obtained at about $0.5 \mathrm{eV}$ by Bos and Lynch [3], Dresselhaus et al. [6] and Tups and Syassen [17]. Thus our peak A at $0.84 \mathrm{eV}$ lies somewhat on the higher energy side as compared to the experimental measurements. On the other hand, it is at the same position as that obtained by Callaway and Laurent [12] using a fully self-consistent $a b$ initio band scheme. Szmulkowicz and Segall [13] have performed an $a b$ initio band calculation of $\mathrm{Al}$ using AugmentedPlane-Waves but to obtain an agreement with experimental data they used a parametrization approach to reproduce parametrized bands. Only then, they could obtain the low energy absorption peak at $0.5 \mathrm{eV}$. Thus, the two self-consistent calculations [12 and the present work] give exactly the same peak position for $\mathbf{A}$. We must add that Hunderi and Nillson [34] have observed weak structures at $0.85 \mathrm{eV}$ and $2.0 \mathrm{eV}$ and also another formation at $2.4 \mathrm{eV}$ which compare well with our peak positions at $0.84,2.0$ and $2.3 \mathrm{eV}$ as indicated in figure 7.

\section{Conclusion.}

The effect of pressure on the optical properties of aluminium, based on the self-consistent $a b$ initio band structures are presented. Without using any adjustable parameter, we obtain the main peak B at different pressures in very good agreement with those observed [17]. The low energy peak $A$ is not in total agreement with the scattered experimental data but it is almost at the same position (for $V / V_{0}=1$ ) as that obtained by Callaway and Laurent [12]. We obtain structures at $2.0 \mathrm{eV}$ and $2.3 \mathrm{eV}$ (Fig. 7) which agree with those observed by Hunderi and Nilsson [34]. It is comforting to note that a selfconsistent $a b$ initio band scheme explains satisfactorily the optical properties of aluminium at different pressures.

\section{Acknowledgments.}

The numerical calculations were performed at CRAY 1 - Palaiseau under a CNRS Research Project and it is a pleasure to acknowledge the help of the staff members of CRAY and MULTICS. The LMTO programme was very kindly provided to us by professor O. K. Andersen and his group at Stuttgart. The authors are very grateful to professors E. Daniel and Dr C. Koenig for very illuminating discussions during completion of this work. 


\section{References}

[1] Ehrenreich, H., Philipp, H. R., Segall, B., Phys. Rev. 132 (1963) 1918.

[2] BennetT, H. E., BenNetee, J. M., in Optical Properties and Electronic Structure of Metals and Alloys, ed. by F. Ablès (North-Holland, Amsterdam) 1966, p. 175.

[3] Bos, L. W., Lynch, D. W., Phys. Rev. Lett. 25 (1970) 156.

[4] Brust, D., Phys. Rev. B 2 (1970) 818.

[5] Ashcroft, N. W., Sturm, K., Phys. Rev. B 3 (1971) 1898 ; ibid 24 (1981) 2315.

[6] Dresselhaus G., Dresselhaus, M. S., Beaglehole, D., Proc. 3rd. Materials Research Symp., NMS Special Publication no 323 (1971) p. 33.

[7] Mathewson, G., Myers, H. P., J. Phys. F : Metal Phys. 2 (1972) 403.

[8] Sturm, K., Ashcroft, N. W., Phys. Rev. B 10 (1974) 1343.

[9] BenBow, R. L., Lynch, D. W., Phys. Rev. B 12 (1975) 5615.

[10] Friedl, C., Ashcroft, N. W., Phys. Rev. B 12 (1975) 5552.

[11] Shiles, E., Sasaki, T., Inokuti, M., Smith, D. Y., Phys. Rev. B 22 (1980) 1612.

[12] Callaway, J., Laurent, D., Phys. Lett. 84A (1981) 499 ;

Singhal, S. P., Callaway, J., Phys. Rev. B 16 (1977) 1744.

[13] Szmulkowicz, F., Segall, B., Phys. Rev. B 24 (1981) 892.

[14] Weaver, J. H., KrafKa, C., Lynch, D. W., Koch, E. E., Optical Properties of Metals (Fachinformationzentrum, Kalsruhe) 1981.

[15] McMahan, A. K., Moriarty, J. A., Phys. Rev. B 27 (1983) 3235.

[16] LaM, P. K., Cohen, M. L., Phys. Rev. B 27 (1983) 5986.

[17] Tups, H., Syassen, K., J. Phys. F : Metal Phys. 14 (1984) 2753.
[18] Andersen, O. K., Phys. Rev. B 12 (1975) 3060 ; ANDERSEN, O. K., JePSen, O., Physica 91B (1977) 317 ; Mackintosh, A. R., ANDERSEN, O. K., Electrons at Fermi Surface, ed. by M. Springford (Cambridge University Press, New York) 1980.

[19] Skriver, H. L., The LMTO Method, Muffin-Tin Orbitals and Electronic Structure, Springer Series in Solid State Sciences 41 (Springer Verlag, Berlin) 1984.

[20] Von Barth, U., Hedin, L., J. Phys. C 5 (1975) 1629.

[21] Levinson, H. J., Greuter, F., Plummer, E. W., Phys. Rev. B 27 (1983) 727.

[22] Nieminen, R. M., Hodges, C. H., J. Phys. F : Metal Phys. 6 (1976) 573.

[23] Pettifor, D. G., Commun. Phys. 1 (1976) 141.

[24] Libernan, D. A., Phys. Rev. B 3 (1971) 2081.

[25] Born, M., Huang, K., Dynamical Theory of Crystal Lattices (Oxford University Press, London) 1956.

[26] JanaK, J. F., Moruzzi, V. L., Williams, A. R., Phys. Rev. B 12 (1975) 1257.

[27] Koenig, C., Khan, M. A., Phys. Rev. B 27 (1983) 6129. Khan, M. A., Koenig, C., Riedinger, R., J. Phys. F : Metal Phys. 13 (1983) L 159.

[28] Lehman, G., TaUt, M., Phys. Status Solidi (b) 54 (1972) 469.

[29] Larson, C. O., Gordon, W. L., Phys. Rev. 156 (1967) 703.

[30] Pearson, W. B., in Handbook of Lattice Spacing and Structure of Metals (Pergamon Press) 1958, p. 313.

[31] Syassen, K., Holzapfel, W. B., J. Appl. Phys. 15 (1978) 1617.

[32] Uspenski, Yu. A., Maksmov, E. G., Rashkeev, S. U., Mazin, I. I., Z. Phys. B 53 (1983) 263.

[33] Alouani, M., Koch, J. M., Khan, M. A., J. Phys. F : Metal Phys. (1985) submitted.

[34] Hunderi, O., Nillson, P. O., Nuovo Cimento B 39 (1977) 459. 